\title{
A solitary jejunal vascular abnormality: a source of massive rectal bleeding
}

\author{
M.P. Saunders \\ The Surgical Unit, Guy's Hospital, London SE1 9RT, UK
}

\begin{abstract}
Summary: Haemorrhage of small bowel origin may prove to be a clinical and diagnostic challenge, particularly when the bleeding is massive and continuous. Such a case is described where the source of blood loss was a jejunal vascular abnormality.
\end{abstract}

\section{Introduction}

Vascular abnormalities of the small intestines are an infrequent though important source of gastrointestinal haemorrhage. ${ }^{1,2}$ Successful management depends primarily upon localizing the source of bleeding, though this is notoriously difficult. ${ }^{3}$ Conventional methods of examination are rarely helpful, the precise site and correct diagnosis often depends upon selective visceral angiography. ${ }^{1,4}$

This is a case report of a 34 year old man with a solitary vascular abnormality of the jejunum causing massive rectal bleeding. The lesion described shows similar clinical features to reported arteriovenous malformations of the small intestines. ${ }^{4,5} \mathrm{~A}$ precipitating cause for the haemorrhage is suggested.

\section{Case report}

A 34 year old male presented complaining of a single episode of rectal bleeding. Whilst waiting to be assessed he collapsed due to a massive gastrointestinal haemorrhage.

Six hours earlier he had suffered colicky abdominal pains followed by diarrhoea and vomiting. There was no haematemesis. He described passing both fresh and altered blood mixed in with liquid stool per rectum on one occasion. There was no relevant past medical history, no family history of gastrointestinal pathology nor bleeding disorders. On direct questioning he denied dyspeptic symptoms and there was no history of altered stool to suggest previous episodes of rectal bleeding. He did

Correspondence: M.P. Saunders, B.Sc., F.R.C.S.

Accepted: 4 March 1991 not take regular medication though had recently self-prescribed ibuprofen, a non-steroidal antiinflammatory agent, for headache.

At initial examination he was clinically shocked. There were no stigmata of chronic liver disease nor cutaneous vascular abnormalities. His abdomen was palpably normal and rectal examination confirmed the presence of altered blood. Despite active resuscitative measures over the following 2 hours his condition remained unstable and his vital signs (rising pulse, falling blood and central venous pressures) indicated presistent active bleeding.

An oesphago-gastro-duodenoscopy was normal. In line with this hospital's radiological department policy a ${ }^{99} \mathrm{Tc}$-labelled red blood cell scan preceded a selective visceral angiogram. The radionucleotide scan was rapidly positive identifying the bleeding site in the proximal small intestine. A selective superior mesenteric arteriogram confirmed this and identified a solitary jejunal bleeding lesion (Figure 1).

At laparotomy there was a $1 \mathrm{~cm}$ firm polypoid lesion on the mesenteric border of the proximal jejunum corresponding to the site of extravasation of contrast (Figure 1). The adjacent intestine and mesentery were normal. The laparotomy was otherwise unremarkable apart from altered blood within the lumen of almost the entire length of intestine. A wedge resection of the jejunum was performed with an end-to-end two layers anastomosis.

Although his post-operative recovery was uneventful the initial management was dominated by the secondary manifestations of massive fluid exchange. Within the course of 24 hours, from the commencement of his symptoms he had received 22 units of blood products, 6 units of synthetic colloid and 2.5 litres of crystalloid fluids.

Microscopic examination revealed the polypoid lesion to be largely organized thrombus (Figure 2). 


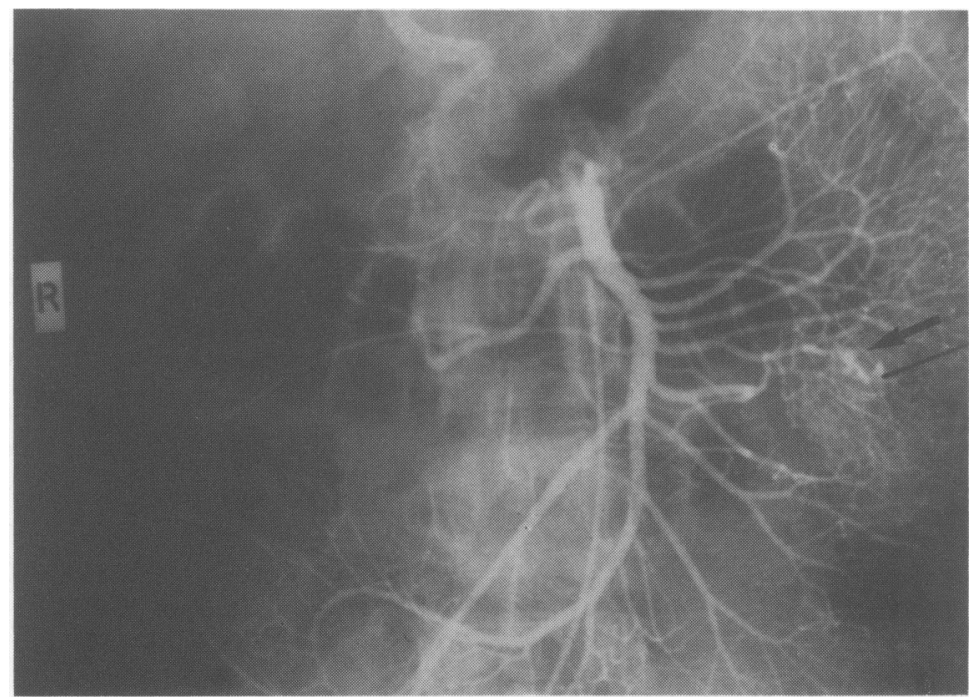

Figure 1 Selective (SMA) angiogram showing extravasation of contrast arrow from a solitary lesion in the proximal jejunum (2 seconds after injection).

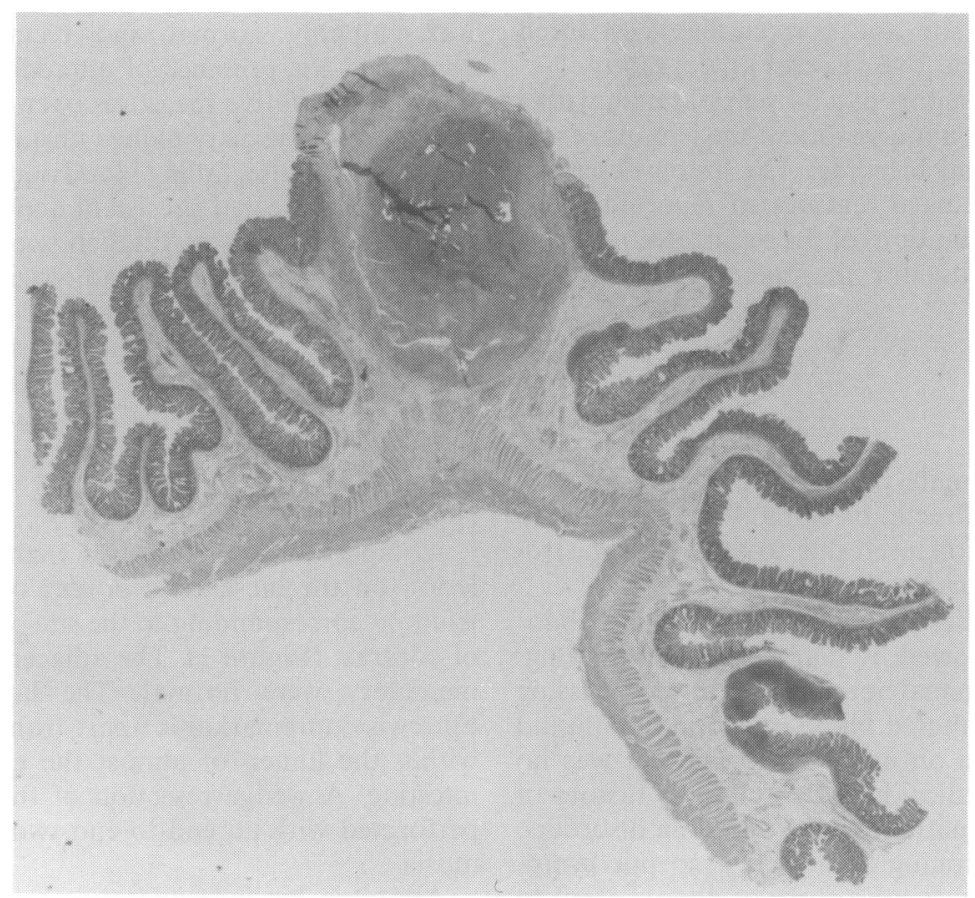

Figure 2 Longitudinal section (haematoxylin and eosin staining) of resected jejunum containing 'polypoid' lesion (original magnification $\times 8$ ).

It was, however, associated with a large solitary submucosal jejunal artery. Detailed histopathology and special staining techniques confirmed a normal arterial wall though its increased diameter was disproportionate to surrounding jejunal vasculature (Figure 3). A congenital origin for this abnormal artery is proposed although the mechanism for rupture could not be elucidated. 


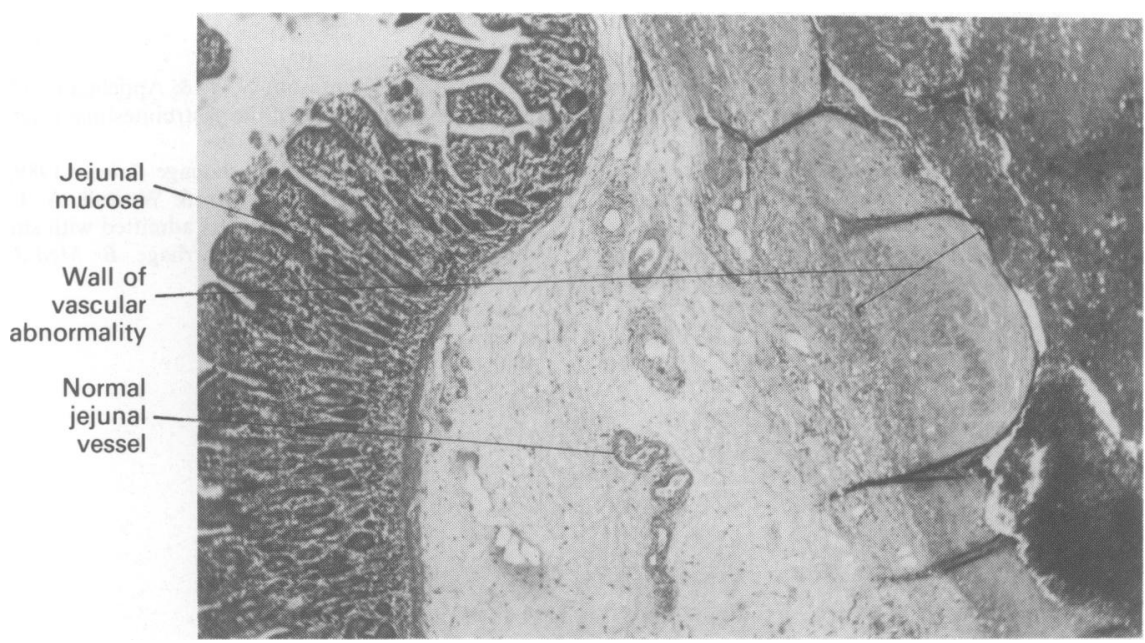

Figure 3 Longitudinal section (haematoxylin and eosin staining) demonstrating the wall of the massive submucosal jejunal artery. Normal vessels are present for comparison (original magnification $\times 120$ ).

\section{Discussion}

The scarcity of reported cases of small intestinal vascular abnormalities presenting with acute gastrointestinal haemorrhage may reflect a lack of recognition, or perhaps confusion due to the variety of descriptive terms used, rather than a true incidence. Haemorrhage traced ultimately to a small bowel origin often proves difficult to localize and requires a disproportionate amount of time and resources. ${ }^{2,3}$ Attributable factors include its length, mobility and relative inaccessability.

From the hospital records of 406 patients admitted with gastrointestinal haemorrhage, initially of unknown cause, 26 were attributable to duodenal and small bowel pathology. Despite extensive investigations 17 were ultimately discharged without an identifiable lesion. The remaining 9 patients had arteriovenous malformations though specifically only one case was identified in the jejunum and that only at histopathology. ${ }^{1}$ In a retrospective review, over a 10 year period, all cases of obscure gastrointestinal bleeding traced to a small bowel origin were recorded by Thompson et al. ${ }^{2}$ This highly selective series provides a useful differential diagnosis. Of the 37 cases collected, 13 were vascular abnormalities, but little more than half could be identified histologically.

A proximal small intestinal site is recorded in 8 further cases from a series of 37 bleeding vascular malformations of the intestines. ${ }^{4}$

In a classification of intestinal arteriovenous malformations Moore et al. describe 3 clinical types. ${ }^{5}$ Type 2 lesions, which most closely resemble the vascular abnormality described in this case report, arise in younger patients (less than 50 years old), they are larger and more easily visible and are commonly located in the small intestines. Similar clinical features coupled with angiographic evidence of an early draining vein led Richardson $e t$ $a l .{ }^{4}$ to speculate that 'Type 2' lesions represent true arteriovenous malformations and are likely to be of congenital origin. At a microscopic level few similarities exist since this lesion has neither supporting venous lakes nor complementary areas of angiodysplasia commonly described in arteriovenous malformations. ${ }^{4,5}$ The solitary 'giant' jejunal artery described in this case report fails to conform to any of the previously described vascular malformations $s^{1,2,4,5}$ though it too is most likely of congenital origin.

The cause of haemorrhage may have been related to the patient's recent ingestion of non-steroidal anti-inflammatory agents. An association between intestinal mucosal damage and these drugs has been reported ${ }^{6}$ and these patients are at greater risk of intestinal haemorrhage and perforation. ${ }^{7}$

\section{Acknowledgement}

I would like to thank Mr M.H. Jourdan, Ph.D., M.S., F.R.C.S., Senior Lecturer and Consultant Surgeon, Guy's Hospital, for his help in the preparation of this manuscript and for permitting me to report this case. 


\section{References}

1. Monk, J.E., Smith, B.A. \& O'Leary, J.P. Arteriovenous malformations of the small intestine. South Med J 1979, 82: 18-22.

2. Thompson, J.N., Hemingway, A.P., McPherson, G.A.D. et al. Obscure gastrointestinal haemorrhage of small bowel origin. Br Med J 1984, 288: 1663-1665.

3. Spiller, R.C. \& Parkins, R.A. Recurrent gastrointestinal bleeding of obscure origin: report of 17 cases and a guide to logical management. Br J Surg 1983, 70: 489-493.

4. Richardson, J.D., Max, M.H., Flint, L.M. et al. Bleeding vascular malformations of the intestines. Surgery 1978, 84: 430-436.
5. Moore, J.D., Thompson, N.W. \& Appelman, H.D. Arteriovenous malformations of the gastrointestinal tract. Arch Surg 1976, 111: 381-389.

6. Editorial, NSAIDs and gut damage. Lancet 1989, ii: 600 .

7. Langman, M.J.S., Morgan, L. \& Worrall, A. Use of antiinflammatory drugs by patients admitted with small or large bowel perforations and haemorrhage. $\mathrm{Br} M e d J$ 1985, 290: $347-350$. 